\title{
Effects of Reference Price and Collaborative Product Promotion on Product Innovation in Supply Chain
}

\author{
Baojun Zhang, Shaojian Qu, and Panpan Li
}

\begin{abstract}
Modern products such as smart phones are quickly updated with limited innovation. However, the promotion level of these products is very high in the market. Literature has not adequately addressed this phenomenon above. This paper studies it from consumer behavior and the collaboration of the supply chain. The model of this paper integrates firms' innovation and promotion decisions in a two-tier supply chain, where a monopoly manufacturer sells products to end-customers through a distributor. The level of product renewal and the level of promotion are decided by the manufacturer and the dealer respectively. Research shows that, when the proportion of marginal revenue between the manufacturer and the distributor meets certain conditions, the game has a unique equilibrium. The manufacturer has the only optimal level of product innovation. In addition, if the manufacturer chooses the best participation rate with the distributor to carry out the product promotion, the profits of both sides are increased. We can see that the collaboration of the supply chain indirectly promotes the limited product updates. The conclusions of the study have some guiding significance for the product innovation and promotion.
\end{abstract}

Index Terms-Innovation, promotion, collaboration, consumer behavior.

\section{INTRODUCTION}

Iphone is an intelligent mobile phone series developed by the Apple Corp in the United States. It positions itself in the middle and upper end of the market. Iphone releases new products every year, and its' products are very popular with consumers. We find that the product innovation is limited between two generations. However, the promotion of new products has not been reduced by comparing the product development expenditure and marketing expenditure of the Apple Corp.

Then we have the following questions: Why is the level of product renewal limited with the increase of product research and development (R\&D) budgets? Why do manufacturers have such a high enthusiasm for product promotion? What is the mechanism that drives enterprises to make such decisions? This paper tries to explain the above questions.

The survival of enterprises depends on the product, and if enterprises want to have a better development, it depends on product innovation. Innovation is regarded as a method to gain competitive advantage and to ensure longstanding tenacity of the enterprise. As we all know, R\&D is a strong support for innovation which is anchored in. $R \& D$ is one of

Manuscript received June 9, 2018; revised August 12, 2018. This work was supported in part by the National Social Science Foundation of China (Grant No. 17BGL083).

The authors are with the Business School, University of Shanghai for Science and Technology, Shanghai, 200093, P.R China (e-mail: jun920802@126.com, qushaojian@usst.edu.cn, panpan7819@126.com). the strategic cores of enterprises' survival and development. Usually, a company's R\&D effort is characterized by lower cost [1] and increased demand [2]. Successful innovation should contribute to improving the firm performance via focusing on such aspects of products such as quality and the brand image, which in turn are thought of as important criteria for customer purchasing decisions [3]. Therefore, the research of product innovation activities has been paid more attention by scholars and members of the supply chain.

Facing increasingly fierce market competition, enterprises pay attention to the management of supply chain on the bas is of their own internal management. Corporate advertising investment strategy is another important content in supply chain management. The members of the supply chain enterprises by advertising accumulate goodwill and obtain a favorable position in the fierce competition in the market. This phenomenon has attracted many domestic, foreign industry and academia's attention, as a study focus. Manufacturers and retailers determine the level of investment in advertising and product categories through mutual coordination and cooperation. They optimize the supply chain decisions, moreover improve the performance of the supply chain members [4].

In addition, studies on behavioral factors have found that the behavior of consumers is affected by the anchoring effect [5]. Part icularly, customers often develop their o wn ideas of a "fair price", also referred to as the reference price, after observing past prices of the product. Nu merous studies have shown that the impact of reference prices on consumer purchase behavior is significant [4], [6]-[12].

In this study, we consider a supply chain that includes a monopoly manufacturer and an independent distributor, where the monopoly manufacturer sells to the ultimate consumers through the distributor. Both the innovation effort and the promotion investment will contribute to the demand of the ultimate consumers. In view of the importance of the reference price effect, our model combines product innovation and product promotion with the reference price effect. We seek the best level of innovation and promotion to achieve the maximum benefits of these two enterprises. Through the above analysis, we will exp lain why the product innovation in the market is limited. In addition, under limited product innovation constraints, we explore whether the manufacturer's participation in product promotion has contributed to the earnings of the two companies. And in the whole process, this paper analyzes the influence of the reference price effect on the above decision.

The rest of this paper is structured as follows. Section II reviews the related literature. Section III specifies the model. Section IV solves the three-stage game to obtain their decision on product innovation, product promotion and 
promotion participation. Section $\mathrm{V}$ conducts numerical analysis. Section VI concludes the work.

\section{LITERATURE REVIEW}

There are two streams of literature which are relevant to our work. The first stream of literature deals with the product innovation and supply chain collaboration. The second deals with the reference price effect.

The research on product innovation management mainly focuses on five points: 1) Research on strategy and evaluation theory of new product [13]; 2) Research on the success and failure of new product [14]; 3) Research on development performance of product [15]; 4) Research on innovation organization theory [16]; 5) Research on cost of design function for product [17].

Anthony [18] interprets collaboration as two or more companies that share the responsibility of co-planning, managing, executing, and measuring performance information. Collaboration usually plays an important role in the business decision making. Generally speaking, innovation collaboration is mainly divided into two categories: horizontal collaboration and vertical collaboration [19]-[22]. However, there is little research on collaborative promotion. This article innovatively combines product innovation with collaborative promotion.

Winer [23] builds a model to predict the probability of purchasing a product by a linear function of, among other variables, the difference between the reference price and the observed price. After him, the influence of the reference price has been paid more and more attention by scholars and managers. Nonetheless, according to the literature we have mastered, the impact of reference prices on product innovation and collaborative decision-making is rarely studied.

Closely related to our work, Song et al. [24] first integrate firms' innovation and advertising decisions in a two-echelon supply chain. The study shows that the impact of innovation and advertising on demand will clearly disrupts both the optimal operation and marketing decisions. Further, they assume that manufacturers are more keen on cooperation. However, our paper differ from the above studies in that we set up a dynamic model considering the behavioral factors of consumers. When manufacturers make decisions on the level of updating, they consider not only the cost of updating but also the market factor of consumer behavior. Our model focuses on collaborative promotion and we find a different phenomenon from their conclusions. It is not always beneficial for the manufacturer to participate in product promotion, while the dealer is always keen on collaborative promotion.

We find that many of the products are updated quickly, but in fact the company's new product innovation is very limited. Most of the previous studies explain this phenomenon from the perspective of brand competition [25], [26]. In this paper, we explain the phenomenon from consumer behavior and the collaboration of the supply chain. We study the three-stage game of the supply chain:

1) Whether the manufacturer decides to participate in product promotion, as well as how to set the participation rate.
2) Whether the dealer decides to accept the dealer's subsidies.

3) Both the manufacturer and the dealer make their level decisions simultaneously.

We have taken into account the internal factors of the manufacturer and the external factors of the market to exp lain the phenomenon of limited product innovation. The model integrates product innovation and product promotion to explore other ways for manufacturers to improve the product demand. Further, we explore the impact of reference prices on the optimal operation and marketing decisions.

\section{THE MODEL}

In this paper, the notations are included in Table I. We consider a two-tier supply chain, where a monopoly manufacturer sells products to customers through a distributor. The model of this paper integrates product innovation and product promotion with the effect of reference price in this supply chain. The specific process is shown in Fig. 1. The manufacturer sets the wholesale price $(w)$ at which the product is sold to the retailer and also decides the innovation level of the product $(a)$. The retailer in turn sets the retail price $(p)$ at which it finally markets the developed product and also decides the promotion level of the product $(q)$. In order to maximize its own income, the manufacturer can decide whether to cooperate with the dealer for product promotion. And product innovation and product promotion will affect the demand of the product.

TABLE I: NOTATION

\begin{tabular}{ll}
\hline \hline Notation & Explanation \\
\hline$a(t)$ & innovation level of the manufacturer \\
$q(t)$ & promotion level of the distributor \\
$C_{a}$ & the innovation costs \\
$C_{q}$ & the promotion costs \\
$c$ & the basic cost of the product \\
$w$ & the wholesale price of the product \\
$p$ & the price of the product \\
$T$ & R\&D time of unit innovation level \\
$G(t)$ & the consumers' purchase intention \\
$r(t)$ & the consumers' reference price \\
$S(t)$ & the demand \\
$\varepsilon$ & manufacturers' participation rate in promotion \\
$\rho$ & the discount rate \\
\hline \hline
\end{tabular}

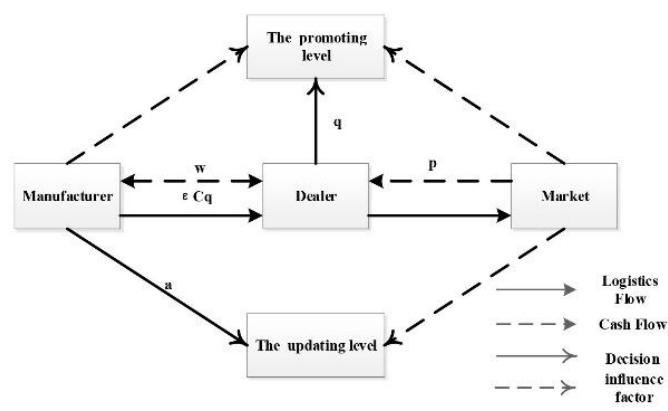

Fig. 1. The process.

The update level of the product is determined by the manufacturer, hence, the update cost involves the expense of product development and the expense of invoking the resources to achieve this update. Refer to previous studies, 
we characterize the cost of product updates as follows [21], [27]. The formula contains an upfront investment equal to the fixed cost of investment which is a function of the level of innovation $(a)$ and the variable development cost which is dependent on the time taken to develop the innovation [27].

$$
C_{a}=I a^{2}+\chi a T
$$

Here, $I, \chi, T$ are positive. $I$ is the investment parameter. $\chi$ is a constant and $T$ is the time taken for technology development.

The higher the level of promotion means the higher the cost of promotion. Similar to previous literatures, the advertising costs are assumed quadratic [4], [24], [28], i.e.,

$$
C_{q}=\frac{1}{2} q^{2}
$$

Generally speaking, when consumers find new products have higher level of innovation and promotion, consumers will consider this new product as relatively higher grade. The above views will enhance the consumer's willingness to buy. Usually, if the time interval of a product innovation is too long, it will reduce the consumer's willingness to buy. Referring to the previous research results [28], the state transition equation of purchase intention will satisfy the following framework.

$$
\dot{G}(t)=\theta_{1} a+\theta_{2} q-\lambda a T-\delta G,
$$

here, $G(0)=G_{0}, G_{0}>0$ is the base of purchase intention. $G$ is the accumulated purchase intention over time $t . \theta_{1}, \theta_{2}$ and $\lambda$ are positive constant. If the update level and the promotion level are high, they will promote the consumer's willingness to buy. But longer update time of product will reduce consumers' desire to buy. $\delta>0$ is the diminishing rate of intention.

For the following reasons, we as sume that the price and the wholesale price are constants. First, in our model, if the price is not constant, the dealer will change the sales price frequently. Similarly, if the wholesale price is changed, the manufacturer and the distributor have to negotiate the wholesale price frequently. However, this is not reasonable in the actual sales process of the product. Changing price frequently will affect consumer's positioning of the product brand, that is, frequent discounts will reduce the consumer's reference price for the product [29]. Consequently, both the manufacturer and the distributor will not change the sales price and the wholesale price frequently. Secondly, product renewal and product promotion have been shown through demand, reference effect and purchase intention. The focus of this paper is not the problem of product pricing. Finally, the reality is that, like the Apple Corp, they have the same price for the same series of products (unless the product rank is changed).

The level of product innovation and promotion will also affect the consumer's reference price. Reference price that exists in the minds of consumers is a consumer behavior. In this model, the consumer reference price is not only affected by the past product image, but also influenced by the level of innovation and promotion. When a consumer wants to buy a product, he will compare the price of the product with his own reference price. If $r>p$, consumers buy the products; If $r \leq p$, consumers do not buy the products. The state transition equation of the reference price satisfies the following equation:

$$
\dot{r}(t)=\mu_{1} a+\mu_{2} q+\beta(p-r),
$$

Here, $r(0)=r_{0}, r_{0}$ is the base of reference price. $r$ is the accumulated reference price over time $t \cdot \mu_{1}, \mu_{2}$ are positive constant. If the update level and the promotion level are high, they will raise the reference price that in the consumers' minds. And, $\beta(p-r)$ reflects the past experience of consumers. $\beta>0$, called "memory parameter" [6], [9]. In general, the consumer will buy a product, if he thinks that the reference price of the product is greater than the price of the product. Otherwise, the consumer will not buy the product. Thus, there is $r>p$, i.e. $(p-r)$ is a negative number. A larger value of $\beta$ reflects the consumer's shorter memory of the past. This value also reflects the low loyalty of consumers to the product.

From the above analysis, we can think that the consumer's reference price, purchase intention, the level of innovation and the level of promotion of the product will affect the demand for the product $S$ (over time).

$$
S(t)=\alpha(r-p)+G+d a+\phi q
$$

Here, $\alpha, d, \phi$ are positive. $G$ indicates that high consumer purchase will directly increase the demand for products. Since $d, \phi$ are positive, the level of innovation and promotion will have a positive impact on product demand. $(r-p)$ indicates the impact of reference prices on demand: when $r>p$, it has a positive impact on demand. When $r<p$, it has a negative impact on demand. A higher $\alpha$ explains consumers are more sensitive to the gap between their reference price and the product price.

Whether the manufacturer decides to participate in the promotion activities. Assuming that the manufacturer's participation in the promotional activities is $\varepsilon$, i.e. the manufacturer subsidizes part of R\&D expenses to the dealer. So, the dealer will improve the level of product promotion. At this point, we can establish the manufacturer's revenue function:

$$
\Pi_{M}(t)=(w-c) S-I a^{2}-\chi a T-\frac{1}{2} \varepsilon q^{2} .
$$

The dealer's revenue function is:

$$
\Pi_{R}(t)=(p-w) S-\frac{1}{2}(1-\varepsilon) q^{2} .
$$

The degree of participation in the promotion of the product does not depend on the time. The reasons are as follows: first, the strategic communication between manufacturers and distributors involves many factors. So participation will not change frequently. Secondly, the focus of our study is whether there is a degree of participation, this participation will increase the profits of manufacturers and distributors. 
Finally, assuming that the degree of participation does not vary with time, we can simplify the model.

The purpose of the manufacturer is to get the best level of product innovation and participation of the product promotion. In addition, the purpose of the dealer is to get the best level of product promotion. Therefore, they can achieve the respective maximum present values of their profits respectively, i.e.,

$$
\begin{aligned}
& \max _{a} J_{M}=\int_{0}^{+\infty} e^{-\rho t}\left((w-c) S-I a^{2}-\chi a T-\frac{1}{2} \varepsilon q^{2}\right) d t \\
& \max _{q} J_{R}=\int_{0}^{+\infty} e^{-\rho t}\left((p-w) S-\frac{1}{2}(1-\varepsilon) q^{2}\right) d t
\end{aligned}
$$

The marg inal utility of innovation will decrease in the level of innovation. And taking into account the cost factors, we believe that manufacturers will not have unlimited increase in the level of product innovation. Similarly, the dealer's product promotion level is not infinite. Thus we get: $0 \leq a(t), q(t) \leq M$, where $M$ is a sufficiently large constant.

\section{ANALYSIS OF THE GAME}

We will study the product promotion Stackelberg game between the manufacturer and the distributor. We can obtain their decision on product innovation, product promotion and promotion participation.

First and foremost, the manufacturer determines the participation rate of the product promotion. When the participation decision is made, the manufacturer and the dealer will determine their own level of effort (manufacturer product update level; dealer - product promotion efforts). Since these decisions may change over time, we can conclude that they make their level decisions simultaneously. We solve the game by backward deduction. In order to solve the Stackelberg game, at first they should set the manufacturer's participation rate $\varepsilon$. We can establish the present value Hamilton function:

$$
H_{M}=(w-c) S-I a^{2}-\chi a T-\frac{1}{2} \varepsilon q^{2}+\gamma_{1 M} \dot{G}(t)+\gamma_{2 M} \dot{r}(t),
$$

$$
H_{R}=(p-w) S-\frac{1}{2}(1-\varepsilon) q^{2}+\gamma_{1 R} \dot{G}(t)+\gamma_{2 R} \dot{r}(t)
$$

Here, $\gamma_{1 M}, \gamma_{2 M}\left(\gamma_{1 R}, \gamma_{2 R}\right)$ represent the state variables of the consumers' purchase intention and the reference price to the manufacturer (the dealer).

Solving the above Hamilton function, we can get the following conclusions. For the proof, please see appendix.

Proposition 1. When the participation decision is made, taking into account the impact of consumer purchase intention and the reference price, in order to achieve the maximization of their own income, the optimal level of product innovation and the level of promotion efforts are:

$$
\bar{a}=\frac{w-c}{2 I}\left(d+\frac{\theta_{1}-\lambda T}{\rho+\delta}+\frac{\alpha \mu_{1}}{\rho+\beta}\right)-\frac{\chi T}{2 I},
$$

$$
\bar{q}=\frac{p-w}{1-\varepsilon}\left(\phi+\frac{\theta_{2}}{\rho+\delta}+\frac{\alpha \mu_{2}}{\rho+\beta}\right) .
$$

From proposition 1, we can see that both the level of product innovation and product promotion are affected by their marg inal revenue. When the memory parameter and the diminishing rate of intention increase, the level of optimal product renewal and the level of promotion will decrease. If the time taken for technology development is very long, the manufacturer should appropriately reduce the level of product renewal. The manufacturer can consider other ways to seize the product market. The manufacturer's promotion coordination with the dealer will affect the level of product promotion. We see that the level of product promotion increases when the manufacturer participates in the product promotion.

In particu lar, the optimal level of product renewal and the optimal promotion level are affected by the reference price effect. Specifically, if consumers are more sensitive to the difference between their reference price and the product price, the optimal product update level and product promotion level should be improved. What enlightens the managers is that it is necessary to consider the consumer behavior factors in the supply chain management. If managers choose to ignore the consumer behavior factors, they will lose some potential markets.

Under the given condition, the manufacturer's update level has a unique optimal value. That is, if the manufacturer chooses a low level of update, the potential customer value is greater than the update cost. In this case, the potential earnings of the manufacturer will be lost. On the contrary, the manufacturer chooses an update level which is higher than the optimal value, then the increase of customer value is insufficient to make up for the cost of renewal. In this case, the choice of the enterprise is also unwise.

Proposition 2. By proposition 1 we know that the optimal level of product innovation and the optimal level of product promotion are both constants. We get the consumer's purchase intention and the reference price over time as follows.

$$
\begin{aligned}
& G(t)=D_{1} e^{-\delta t}+G_{S}, \\
& r(t)=D_{2} e^{-\beta t}+r_{S},
\end{aligned}
$$

Here, $\quad D_{1}=G_{0}-G_{S}, G_{S}=\left(\left(\theta_{1}-\lambda T\right) \bar{a}+\theta_{2} \bar{q}\right) / \delta$ $D_{2}=r_{0}-r_{S}$ and $r_{S}=\left(\mu_{1} \bar{a}+\mu_{2} \bar{q}+\beta \rho\right) / \beta$.

We note that consumer purchase intention and the reference price will reach their steady states $G_{S}$ and $r_{S}$ when $t \rightarrow+\infty$. From the steady state of consumer purchase intention, we find that it is mainly affected by the level of product innovation, the level of product promotion and the product time interval. When the level of product promotion rises, the steady-state value of consumer purchase intention becomes larger. In particular, we note that when $\theta_{1}-\lambda T>0$, the level of product innovation has a positive effect on the steady state of purchase intention. In other 
words, when consumers measure their utility of product innovation and wait time, they think that it is worthwhile wait ing. Moreover, if the other conditions are unchanged, the consumer's purchase intention will be greater when the diminishing rate of intention decreases.

Similarly, we analyze the steady state of the reference price. We find that it is mainly affected by the level of product innovation and the level of product promotion. Moreover, they have positive effects on the steady state of the reference price. We find that when the level of product innovation and promotion are zero, the reference price is equal to the selling price of the product. We also find that the steady-state value of the reference price decreases when the consumer's memory parameter becomes larger. A large $\beta$ value reflects the consumer's shorter memory of the past. This value also reflects consumers' low loyalty to the product.

We also find an interesting phenomenon that the reference price of the consumer is related to the stable price of the product. Specifically, when the price of the product is maintained at a high level, the consumer will increase the reference price of the product. It has a certain guiding significance for the brand effect of the enterprise. If an enterprise wants to place a product as a high-end commodity, he should stabilize the price of the product at a relatively high position. The decision can help consumers to distinguish low-end products.

Proposition 3. We get present values of the manufacturer's profit and the dealer's profit based on the manufacturer's promotional participation rate $\varepsilon$.

$$
\begin{aligned}
& J_{M}=\frac{\alpha(w-c)\left(r_{S}-p\right)}{\rho}+\frac{(w-c) G_{S}}{\rho}+\frac{(w-c) d \bar{a}}{\rho}-\frac{I \bar{a}^{2}}{\rho} \\
& +\frac{(w-c) \phi \bar{q}}{\rho}-\frac{\chi \bar{a} T}{\rho}-\frac{\varepsilon \bar{q}^{2}}{2 \rho}+\frac{D_{1}(w-c)}{\rho+\delta}+\frac{\alpha D_{2}(w-c)}{\rho+\beta} \\
& J_{R}=\frac{\alpha(p-w)\left(r_{S}-p\right)}{\rho}+\frac{(p-w) G_{S}}{\rho}+\frac{(p-w) d \bar{a}}{\rho} \\
& +\frac{(p-w) \phi \bar{q}}{\rho}-\frac{(1-\varepsilon) \bar{q}^{2}}{2 \rho}+\frac{D_{1}(p-w)}{\rho+\delta}+\frac{\alpha D_{2}(p-w)}{\rho+\beta} .
\end{aligned}
$$

If consumers think the reference price is greater than the selling price, the difference between those two prices has a positive effect on present values of the manufacturer's profit and the dealer's profit based on the manufacturer's promotional participation rate $\varepsilon$. Specifically, present values of their profits increase with the difference.

Since the manufacturer can get the present value of his profit for any specific promotional participation rate $\varepsilon$, he can then decide the optimal $\bar{\varepsilon}$ to maximize the present value.

Proposition 4. The manufacturer's optimal participation rate of the product promotion is:

$$
\bar{\varepsilon}= \begin{cases}\frac{3 w-2 c-p}{w-2 c+p}, & \text { if } 3 w-2 c>p, \\ 0, & \text { else. }\end{cases}
$$

We can see that when the marginal revenue of the manufacturer and the dealer satisfy $3 w-2 c>p$, the manufacturer's product promotion participation rate will be
$(3 w-2 c-p) /(w-2 c+p)$, and the manufacturer has optimal profit. Interestingly, the manufacturer's optimal participation rate for collaborative promotion is not affected by the reference price effect. For dealers, they welcome manufacturers to participate in product promotion. While in other conditions, the manufacturer's earnings will be lower than the benefits mentioned above. We can clearly see that when manufacturers choose the optimal level of renewal, it is useful for him to participate in the collaborative promotion with the best participation rate. In the next section we will discuss their benefits.

\section{NUMERICAL EXAMPLE}

In this section, we consider a numerical example to analyze the optimal product update level, promotion level and compare the two firms' profit under different participation rate and reference price effect sensitivity. We refer to the previous research results [4], [21] and [30] and set the values of the parameters involved as $\theta_{1}=0.03$, $\theta_{2}=0.04, \chi=2, \lambda=0.01, I=2, \beta=0.2, T=2$, $\delta=0.1, \mu_{1}=0.02, \mu_{2}=0.04, \rho=0.5, \phi=0.5, d=1$.

If $3 w-2 c \leq p, \varepsilon \leq 0$. However, $\varepsilon$ should be greater than 0 . So, we do not discuss this situation. Without loss of generality, we assume that the sale price of the product $p$ is 30 , the wholesale price $w$ is 20 , the basic cost $c$ is 10 . Based on the above conclusions, we can find the optimal promotion participation rate $\bar{\varepsilon}$ is $1 / 3$. We consider four participation rates respectively are $1 / 3,0,2 / 3$ and $1 / 6.1 / 3$ indicates that the participation rate is the best decision, 0 shows that the manufacturer do not participate in collaborative product promotion, 2/3 says that the rate of participation is greater than the optimal decision, $1 / 6$ expresses that the participation rate is less than the optimal decision.

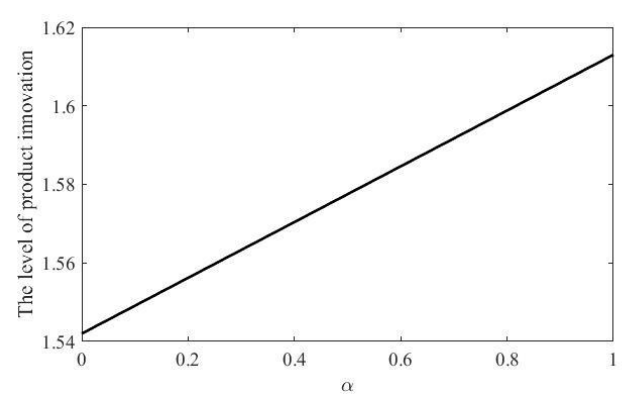

Fig. 2. The level of product innovation.

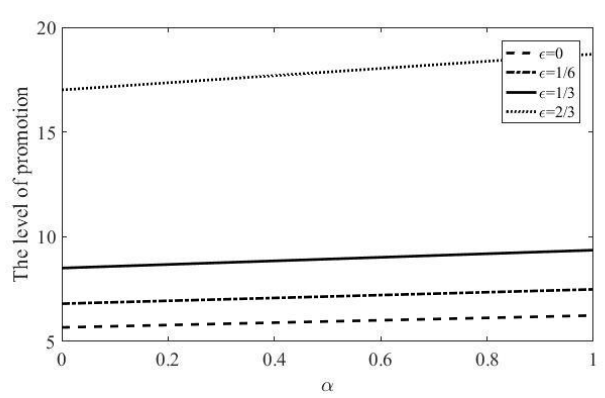

Fig. 3. The level of promotion.

From Fig. 2 and Fig. 3, we have verified some of the explanations from proposition 1 . If consumers are more 
sensitive to the difference between their reference price and the product price, the optimal product update level and product promotion level should be improved. And the optimal level of product promotion is improved with the increase of the manufacturer's participation rate.

We compare the benefits of the manufacturer, the dealer, and the entire supply chain in these four cases. Based on the above parameters, we can get the benefits of the manufacturer, the dealer, and the entire supply chain corresponding to different consumer sensitivity to their reference prices. In order to better reflect the change trend of profit accompanied by the effect of the reference price, we extend the axis of the reference price impact strength.

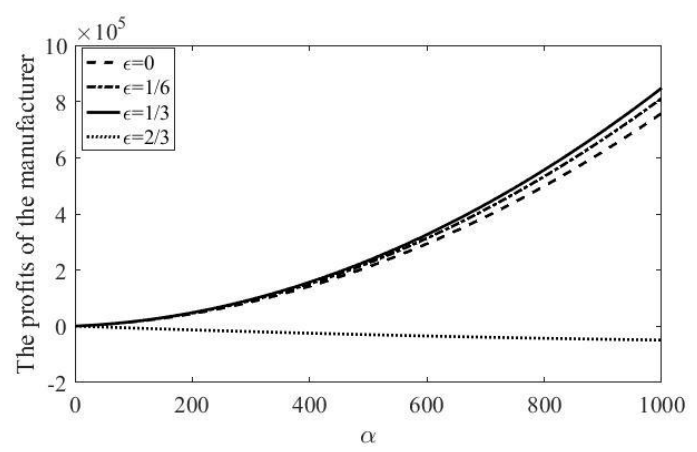

Fig. 4. The profits of the manufacturer.

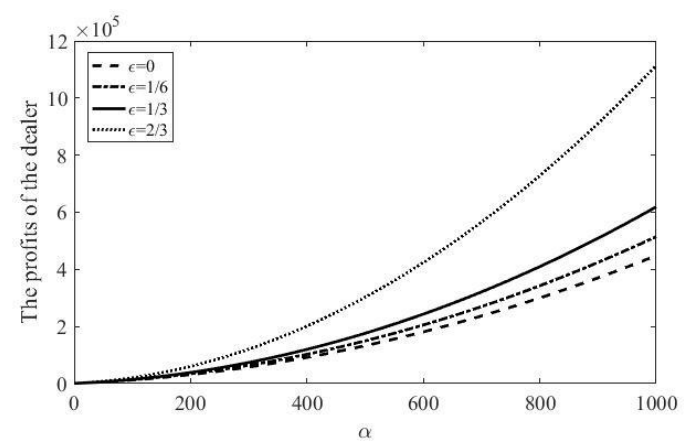

Fig. 5. The profits of the distributor.

As we can see in Fig. 4, when the manufacturer selects the optimal participation rate $1 / 3$, he gains the most. When the manufacturer's participation rate is higher or lower than the optimal participation rate, his earnings are lower than the optimal value. Interestingly, we find that the above phenomena can be divided into two cases. When the participation rate is lower than the optimal value, the profit of the manufacturer is higher than that of the non-participation. On the contrary, when the participation rate is higher than the optimal value, the profit of the manufacturer is lower than that of the non-participation. And with the increase of the impact strength of the reference price, the difference of the above income tends to become larger. In particular, with the increase in the impact of the reference price, the trend of change from the above incomes is different. When the participation rate is greater than the optimal value, the manufacturer's inco me decreases with the greater impact of the reference price. When the participation rate is less than the optimal value, the situation is opposite. It suggests that the manufacturer should be conservative when he chooses to carry out a collaborative product promotion. That is to say, it is always beneficial for the manufacturer to choose a participation rate less than or equal to the optimal value.
Further, the manufacturer should never ignore the reference price effect for the sake of its own interests.

From Fig. 5, when the manufacturer's participation rate increases, the distributor's earnings are increased. That is, the dealer should take a positive attitude towards the collaborative promotion of products. And with the increase in the impact of the reference price, the dealer benefits more from the collaborative product promotion. According to the change of the manufacturer's income, the two enterprises will achieve equilibriu mat the point of the manufacturer's optimal participation rate. Similarly, if the dealer is wise, he would not ignore the reference price effect.

Co mbined with the above findings, they can only reach an agreement that the manufacturer's participation rate is $1 / 3$ and their income will increase under the circumstances. We compare the benefits of the entire supply chain with participation rates of $1 / 3$ and 0 .

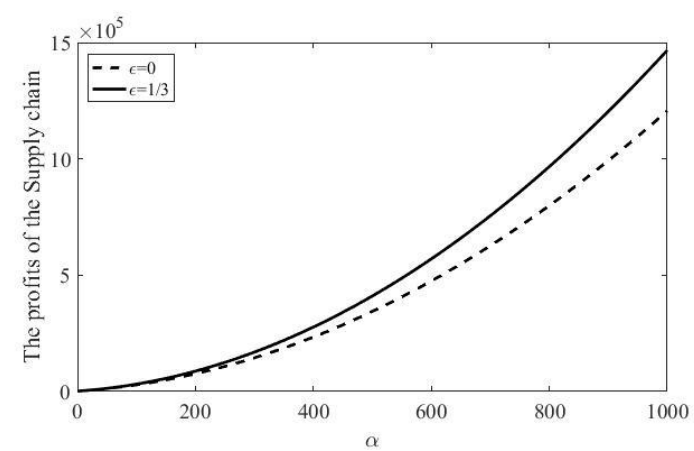

Fig. 6. The profits of the Supply chain

In Fig. 6, we see that the agreement reached by the manufacturer and the dealer is beneficial to the whole supply chain. And with the increase of the impact of the reference price, the equilibrium income of the supply chain is increasing. The results show that the research has some guiding significance for the operation decision of manufacturing industry. Facing the pressure of the cost and market factors, the manufacturer has the best value for the product update level. If the innovation level exceeds this optimal value, his efforts have a negative impact. Whereas, if the manufacturer collaborates with the dealer for product promotion, his earnings will improve. The agreement he reaches with the dealer is beneficial to both parties. The phenomenon above is more obvious in the supply chain with the high impact of the reference price.

\section{CONCLUSIONS}

In this paper, we focused our work on the existing phenomenon in modern products. Although modern products update quickly, its innovation is very limited. However, in spite of the limited level of product renewal, the level of product promotion in the market has not been reduced. We explain this phenomenon from consumer behavior and the collaboration of the supply chain.

We consider a two-echelon supply chain, where a manufacturer sells products to customers through a distributor. The model of this paper integrates product innovation and product promotion with the effect of reference price in this supply chain. The manufacturer and the distributor decide the level of the product renewal and the 
promotion level respectively. It can be found that the manufacturer has an optimal level of renewal due to the double constraints of the cost of renewal and the market factors. When the update level is higher than the optimal value, the manufacturer's earnings will be infringed. This shows that there is an economic explanation for the limited product innovation in the market. However, the manufacturer can promote their own profits through collaborative product promotion. Through our study, we find that when marg inal revenues of the manufacturer and the dealer meet $3 w-2 c>p$, the manufacturer and the dealer will reach an agreement. This protocol is the only optimal solution in this game. The manufacturer's product promotion participation rate is $(3 w-2 c-p) /(w-2 c+p)$, which is responsible for the cost of product promotion $(3 w-2 c-p) /$ $(w-2 c+p)$. Meanwhile, the manufacturer and the dealer can reach an agreement, that is, the manufacturer participates in the collaborative product promotion with his best participation rate. Their benefits are all improved by the agreement.

The model analysis gives some meaningful management inspiration. In the case of the market, it is not necessary for the manufacturer to carry out a high level of product innovation. While ensuring the optimal level of product renewal, the manufacturer should participate in the product promotion with appropriate participation rate to improve its own income. But when the manufacturer decides to participate, he should take a conservative attitude (in other words, he does not accept the participation rate higher the optimal value). At the same time, the dealer should actively communicate with the manufacturer to facilitate the best collaborative promotion. The above analys is also shows that it is very necessary for modern enterprises to pay attention to the promotion of products.

We also find that the influence of the reference price effect on manufacturers and dealers should not be ignored. Specifically, the level of optimal product renewal and the level of promotion are in direct proportion to the reference price effect. In the case of the manufacturer's different participation rate, the reference price effect will amplify the income difference between the two enterprises. Their respective optimal returns also increase with the intensity of the reference price impact. And their respective optimal returns also increase with the reference price effect. It means that as consumers play an increasingly important role in the market, enterprises should attach importance to the reference price effect. Enterprises should pay attention to market research so as to know consumers' voice.

Finally, we want to mention potential extensions for future research. In this paper, we suppose that there is no information asymmetry between the manufacturer and distributor. Firstly, a different focus would be to further the analysis by exploring their optimal strategies with information asymmetry. Secondly, it is interesting to explore their optimal strategies under the assumption that the retailer also implements innovation efforts for the product. Finally, since a bilateral monopoly supply chain is modelled in our paper, it would be interesting to extend a supply chain consisting of more than two firms.

\section{APPENDIX A}

\section{PROOF OF PROPOSITION 1}

From (10), we have: $\partial H_{M} / \partial a=0$, (A.1); $\partial H_{M} / \partial \gamma_{1 M}$ $=\dot{G}$, (A.2); $\partial H_{M} / \partial \gamma_{2 M}=\dot{r}$, (A.3); $\dot{\gamma}_{1 M}=\rho \gamma_{1 M}-\partial H_{M} /$ $\partial G$, (A.4); $\dot{\gamma}_{2 M}=\rho \gamma_{2 M}-\partial H_{M} / \partial r$, (A.5). From (A.1), we have: $\partial H_{M} / \partial a=0$, (A.6). From (A.6), we have: $a=((w-$ $\left.c) d-\chi T+\gamma_{1 M}\left(\theta_{1}-\lambda T\right)+\gamma_{2 M} \mu_{1}\right) / 2 I$, (A.7) and $\gamma_{1 M}\left(\theta_{1}-\right.$ $\lambda T)=2 I a-(w-c) d+\chi T-\gamma_{2 M} \mu_{1}$, (A.8). From (10), we have: $\partial H_{M} / \partial G=w-c-\delta \gamma_{1 M},\left(\right.$ A.9) and $\partial H_{M} / \partial r=\alpha(w$ $-c)-\beta \gamma_{2 M}$, (A.10). Taking (A.9) into (A.4), we have: $\dot{\gamma}_{1 M}=$ $(\rho+\delta) \gamma_{1 M}-(w-c)$, (A.11). Taking (A.10) into (A.5), we have: $\dot{\gamma}_{2 M}=(\rho+\beta) \gamma_{2 M}-\alpha(w-c)$, (A.12). From (A.7), we have: $\dot{a}=\left(\left(\theta_{1}-\lambda T\right) \dot{\gamma}_{1 M}+\mu_{1} \dot{\gamma}_{2 M}\right) / 2 I$, (A.13). Taking (A.11) and (A.12) into (A.13), we have: $\dot{a}=\left(\left(\theta_{1}-\lambda T\right)\left((\rho+\delta) \gamma_{1 M}-\right.\right.$ $\left.(w-c))+\mu_{1}\left((\rho+\beta) \gamma_{2 M}-\alpha(w-c)\right)\right) / 2 I$, (A.14). Taking (A.8) into (A.14), we have: $\dot{a}=\left(\mu_{1}(\beta-\delta) \gamma_{2 M}+(2 I a+\chi T-\right.$ $\left.d(w-c))(\rho+\delta)-\left(\theta_{1}+\alpha \mu_{1}-\lambda T\right)(w-c)\right) / 2 I, \quad(\mathrm{~A} .15)$ and $\mu_{1}(\beta-\delta) \gamma_{2 M}=2 I \dot{a}-(2 I a+\chi T-d(w-c))(\rho+\delta)+\left(\theta_{1}+\alpha \mu_{1}\right.$ $-\lambda T)(w-c),(\mathrm{A} .16)$. From (A.15), we have: $\ddot{a}=(\mu 1(\beta-\delta)$ $((\rho+\beta) \gamma 2 M-\alpha(w-c))+2 I(\rho+\delta) \dot{a}) / 2 I$, (A.17). Taking (A.16) into (A.17), we have: $\ddot{a}=[2 I(2 \rho+\delta+\beta) \dot{a}+(\rho+\beta)$ $\left((\rho+\delta)((w-c) d-2 I a-\chi T)+(w-c)\left(\theta_{1}-\lambda T+\alpha \mu_{1}\right)\right)-$ $\left.\alpha \mu_{1}(\beta-\delta)(w-c)\right] / 2 I,(\mathrm{~A} .18)$. Arranging the above equation, we have: $\quad \ddot{a}-(2 \rho+\delta+\beta) \dot{a}+(\rho+\beta)(\rho+\delta) a=[(\rho+\beta)$ $\left((\rho+\delta)((w-c) d-\chi T)+(w-c)\left(\theta_{1}-\lambda T+\alpha \mu_{1}\right)\right)-\alpha \mu_{1}(\beta-\delta)$ $(w-c)] / 2 I,($ A.19). Solving the above equation, we have: $a(t)=C_{1} e^{(\rho+\beta) t}+C_{2} e^{(\rho+\delta) t}+\bar{a},(\mathrm{~A} .20)$ and $\bar{a}=((w-c)(d+$ $\left.\left.\left(\theta_{1}-\lambda T\right) /(\rho+\delta)+\alpha \mu_{1} /(\rho+\beta)\right)-\chi T\right) / 2 I,($ A.21). Here, $C_{1}$ and $C_{2}$ are unknown parameters. If $C_{i} \neq 0(i=1,2)$ and $t \rightarrow+\infty, a(t)$ will be infinite. Because the level of product innovation can't be infinite in reality, we can get $C_{i}=0(i=1,2)$. Thus $a(t)$ is constant, i.e., when the participation rate of the manufacturer for product promotion is given, the manufacturer's optimal level of product innovation is $\bar{a}$.

The solving process of the optimal product promotion level is similar to the above process. Here we o mit the solving process of the optimal product promotion level.

\section{APPENDIX B}

\section{PROOF OF PROPOSITION 2}

By proposition 1 we know that the optimal level of product innovation and the optimal level of product promotion are both constants. By putting $\bar{a}, \bar{q}$ into (5) and (6), we can replace $a(t)$ to $\bar{a}$ and $q(t)$ to $\bar{q} \cdot d G / d t=\left(\theta_{1}-\lambda T\right) \bar{a}+\theta_{2} \bar{q}$ 
$-\delta G$, (B.1). From (B.1), we have: $\dot{G}+\delta G=\left(\theta_{1}-\lambda T\right) \bar{a}+\theta_{2} \bar{q}$, (B.2). It is similar to $y^{\prime}+p(x) y=q(x)$. Solving the above equation, we can get: $G(t)=e^{-\delta t}\left[D_{1}+\int\left(\left(\theta_{1}-\lambda T\right) \bar{a}+\theta_{2} \bar{q}\right) e^{\delta t}\right.$ $d t]=D_{1} e^{-\delta t}+\left(\left(\theta_{1}-\lambda T\right) \bar{a}+\theta_{2} \bar{q}\right) / \delta,($ B.3). When $\mathrm{t}=0$, we know $G(0)=G_{0}$. We can get: $D_{1}=G_{0}-\left(\left(\theta_{1}-\lambda T\right) \bar{a}+\theta_{2} \bar{q}\right)$ $/ \delta$, (B.4). Let $G_{S}=\left(\left(\theta_{1}-\lambda T\right) \bar{a}+\theta_{2} \bar{q}\right) / \delta$. So, $D_{1}=G_{0}-$ $G_{S}, G(t)=D_{1} e^{-\delta t}+G_{S}$.

The solving process of the reference price over time is similar to the above process. Here we omit the solving process of the reference price over time.

\section{APPENDIX C}

\section{PROOF OF PROPOSITION 3}

Taking $G(t), r(t), \bar{a}$ and $\bar{q}$ into (8), we can get : $\max _{a, q} J_{M}=\int_{0}^{+\infty} e^{-\rho t}\left((w-c) S-I \bar{a}^{2}-\chi T \bar{a}-\varepsilon \bar{q}^{2} / 2\right) d t=$ $\int_{0}^{+\infty} e^{-\rho t}\left((w-c)(\alpha(r-p)+G+d \bar{a}+\phi \bar{q})-I \bar{a}^{2}-\chi T \bar{a}-\frac{1}{2} \varepsilon \bar{q}^{2}\right) d t$ $=\int_{0}^{+\infty} e^{-\rho t}\left((w-c)\left(\alpha\left(D_{2} e^{-\beta t}+r_{s}-p\right)+D_{1} e^{-\delta t}+G_{s}+d \bar{a}+\phi \bar{q}\right)-\right.$ $\left.I \bar{a}^{2}-\chi T \bar{a}-\varepsilon \bar{q}^{2} / 2\right) d t$, (C.1). From (C.1), we have: $J_{M}=$ $\frac{\alpha(w-c)\left(r_{S}-p\right)}{\rho}+\frac{(w-c) G_{S}}{\rho}+\frac{(w-c)(d \bar{a}+\phi \bar{q})}{\rho}-\frac{\bar{a}^{2}}{\rho}-\frac{\chi T \bar{a}}{\rho}-\frac{\varepsilon \bar{q}^{2}}{\rho}+$ $\frac{D_{1}(w-c)}{\rho+\delta}+\frac{\alpha D_{2}(w-c)}{\rho+\beta},(\mathrm{C} .2)$.

The solving process of $J_{R}$ is similar to the above process. Here we omit the solving process of $J_{R}$.

\section{APPENDIX D}

\section{PROOF OF PROPOSITION 4}

Here we solve the manufacturer's optimal participation rate of the product promotion in order to maximize its interests.

From (C.2), we have: $\frac{d J_{M}}{d \varepsilon}=\frac{1}{\rho}\left[(w-c)\left(\frac{\alpha \mu_{2} \bar{q}}{\beta(1-\varepsilon)}+\frac{\theta_{2} \bar{q}}{\delta(1-\varepsilon)}+\right.\right.$ $\left.\left.\frac{\phi \bar{q}}{1-\varepsilon}\right)-\frac{1}{2} \bar{q}^{2}-\frac{\varepsilon \bar{q}^{2}}{1-\varepsilon}\right]-\frac{(w-c) \theta_{2} \bar{q}}{\delta(1-\varepsilon)(\rho+\delta)}-\frac{\alpha(w-c) \mu_{2} \bar{q}}{\beta(1-\varepsilon)(\rho+\beta)}=0$

From (D.1), we have: $\frac{\alpha(\rho+\beta)(w-c) \mu_{2} \bar{q}}{\rho \beta(1-\varepsilon)(\rho+\beta)}-\frac{\alpha \rho(w-c) \mu_{2} \bar{q}}{\rho \beta(1-\varepsilon)(\rho+\beta)}=$ $\frac{\alpha(w-c) \mu_{2} \bar{q}}{\rho(1-\varepsilon)(\rho+\beta)}$, (D.2) and $\frac{(\rho+\delta)(w-c) \theta_{2} \bar{q}}{\rho \delta(1-\varepsilon)(\rho+\delta)}-\frac{\rho(w-c) \theta_{2} \bar{q}}{\rho \delta(1-\varepsilon)(\rho+\delta)}$ $=\frac{(w-c) \theta_{2} \bar{q}}{\rho(1-\varepsilon)(\rho+\delta)},(D .3)$. Taking (D.2) and (D.3) into (D.1), we can get: $\frac{\alpha(w-c) \mu_{2} \bar{q}}{\rho(1-\varepsilon)(\rho+\beta)}+\frac{(w-c) \theta_{2} \bar{q}}{\rho(1-\varepsilon)(\rho+\delta)}-\frac{(1+\varepsilon) \bar{q}^{2}}{2 \rho(1-\varepsilon)}+\frac{(w-c) \phi \bar{q}}{\rho(1-\varepsilon)}=0$, (D.4). From (D.4), we have: $(w-c)\left[\alpha \mu_{2}(\rho+\delta)+\theta_{2}(\rho+\beta)+\right.$ $\phi(\rho+\beta)(\rho+\delta)]=(1+\varepsilon)(\rho+\beta)(\rho+\delta) \bar{q} / 2,(\mathrm{D} .5)$. Taking $\bar{q}$ into (D.5), we can get: $\frac{1}{2}(1+\varepsilon)(\rho+\beta)(\rho+\delta) \bar{q}=\frac{(p-w)(1+\varepsilon)}{2(1-\varepsilon)}\left[\alpha \mu_{2}\right.$. $\left.(\rho+\delta)+\theta_{2}(\rho+\beta)+\phi(\rho+\beta)(\rho+\delta)\right]$, (D.6). From (D.5) and (D.6), we have: $(w-c)\left[\alpha \mu_{2}(\rho+\delta)+\theta_{2}(\rho+\beta)+\phi(\rho+\beta)(\rho+\delta)\right]=$ $\frac{(p-w)(1+\varepsilon)}{2(1-\varepsilon)}\left[\alpha \mu_{2}(\rho+\delta)+\theta_{2}(\rho+\beta)+\phi(\rho+\beta)(\rho+\delta)\right]$,

From (D.7), we can get: $w-c=\frac{(p-w)(1+\varepsilon)}{2(1-\varepsilon)}$,

Arranging the above equation, we can get: $\varepsilon=\frac{3 w-2 c-p}{w-2 c+p}$,

(D.9). So, we can get: $\bar{\varepsilon}= \begin{cases}\frac{3 w-2 c-p}{w-2 c+p}, & \text { if } 3 w-2 c>p \text {, } \\ 0, & \text { else. }\end{cases}$ (D.10).

\section{REFERENCES}

[1] R. Amir, I. Evstigneev, and J. Wooders, "Noncooperative versus cooperative R\&D with endogenous spillover rates," Games and Economic Behavior, vol. 42, no. 2, pp. 183-207, Feb. 2003.

[2] S. M. Gilbert and V. Cvsa, "Strat egic commitment to price to stimulate downstream innovation in a supply chain," European Joumal of Operational Research, vol. 150, no. 3,pp.617-639, Nov. 2003.

[3] M. Karlsson, L. Trygg, and B. O. Elfstrom, "Measuring R\&D productivity: Complementing the picture by focusing on research activities," Technovation, vol. 24, no. 3, pp. 179-186, Mar. 2004.

[4] J. Zhang, Q. Gou, L. Liang, and Z. Huang, "Supply chain coordination through cooperative advertising with reference price effect," Omega-Intemational Journal of Management Science, vol. 41, no. 2, pp. 345-353, Apr. 2013.

[5] G. Kalyanaram and R. S. Winer, "Empirical generalizations from reference price research," Marketing Science, vol. 14, no. 3_supplement, pp. 161-161,1995.

[6] X. Chen, P. Hu, S. Shum, and Y. Zhang, "Dynamic stochastic inventory management with reference price effects," Operations Research, vol.64, no.6, pp. 1529-1536, Nov.-Dec. 2016.

[7] G. Fibich, O. Lowengart, and A. Gavious, "Explicit solutions of optimization models and differential games with nonsmooth (asymmetric) reference-price effects," Operations Research, vol. 51, no. 5, pp. 721-734, Sep.-Oct. 2003.

[8] E. A. Greenleaf, "The impact of reference price effects on the profitability of price promotions," Marketing Science, vol. 14, no. 1, pp. 82-104, 1995.

[9] Z. Hu, X. Chen, and P. Hu, "Technical note-dynamic pricing with gain-seeking reference price effects," Operations Research, vol. 64, no. 1, pp. 150-157, Jan.-Feb. 2016.

[10] P. K. Kopalle, A. G. Rao, and J. L. Assunção, "Asymmetric reference price effects and dynamic pricing policies," Marketing Science, vol. 15, no. 1 , pp. $60-85,1996$.

[11] J. Nasiry and I. Popescu, "Dynamic pricing with loss-averse consumers and peak-end anchoring," Operations Research, vol. 59, no. 6, pp. 1361-1368, Nov.-Dec. 2011.

[12] I. Popescu and Y. Wu, "Dynamic pricing strategies with reference effects," Operations Research, vol. 55, no. 3, pp. 413-429, May.-Jun. 2007.

[13] Y. Hsu, "Relationships between product development strategies and product design issues," Journal of Engineering Design, vol. 22, no. 6, pp. 407-426, 2011.

[14] R. Balachandra, "An expert system for new product development projects," Industrial Managementand Data Systems, vol. 100, no. 7, pp. 317-324, 2000.

[15] X. Koufteros, G. Lu, R. C. Peters, K. H. Lai, C. W. Y. Wong, and T. C. E. Cheng, "Product development practices, manufacturing practices, and performance: A mediat ional perspective," Intemational Journal of Production Economics, vol. 156, pp. 83-97, Oct. 2014.

[16] J. H. Love and S. Roper, "Organizing innovation: Complementarities between cross-functional teams," Technovation, vol. 29, no. 3, pp. 192-203, Mar. 2009.

[17] H. Lee and J. Lee, " Optimal cost estimation for improvement of product design," Intemational Journal of Industrial Engineering-Theory Applications and Practice, vol. 18, no. 5, pp. 232-243, 2011.

[18] P. L. Anthony, H. B. Crawley, P. A. Fischer, R. L. McKay, and W. T. Meyer, "CANbus and microcontroller use in the BaBar detector at 
SLAC," IEEE Transactions on Nuclear Science, vol. 47, no. 2, pp. 166-169, Apr. 2000.

[19] R. Dominguez, S. Cannella, and J. M. Framinan, "The impact of the supply chain structure on bullwhip effect," Applied Mathematical Modelling, vol. 39, no. 23-24, pp. 7309-7325, Dec. 2015.

[20] P. A. Geroski, "Vertical relations between firms and industrial policy," Economic Journal, vol. 102, no. 410, pp. 138-147, Jan. 1992.

[21] A. Jha, K. Fernandes, Y. Xiong, J. Nie, N. Agarwal, and M. K. Tiwari, "Effects of demand forecast and resource sharing on collaborative new product development in supply chain," Intemational Joumal of Production Economics, vol. 193,pp. 207-221, Nov. 2017.

[22] M. Skippari, M. Laukkanen, and J. Salo, "Cognitive barriers to collaborative innovation generation in supply chain relationships," Industrial Marketing Management, vol. 62,pp. 108-17, Apr. 2017.

[23] R. S. Winer, "A reference price model of brand choice for frequently purchased products," Joumal of Consumer Research, vol. 13, no. 2, pp. 250-256, 1986.

[24] J. Song, F. Li, D. D. Wu, L. Liang, and A. Dolgui, "Supply chain coordination through integration of innovation effort and advertising support," Applied Mathematical Modelling, vol. 49, pp. 108-123, Sep. 2017.

[25] Y. Chen, Y. Wang, S. Nevo, J. Benitez-Amado, and G. Kou, "IT capabilities and product innovation performance: The roles of corporate entrepreneurship and competitive intensity," Information \& Management, vol.52, no. 6, pp. 643-657, Sep. 2015.

[26] W. Tian, "Supply chain coordination of competitive retailers' innovat ion investment," Computer Integrated Manufacturing Systems, vol. 15, no. 1, pp. 147-52, Jan. 2009.

[27] S. R. Bhaskaran and V. Krishnan, "Effort, revenue, and cost sharing mechanisms for collaborative new product development," Management Science, vol. 55, no. 7, pp. 1152-1169, Jul. 2009.

[28] S. Jørgensen, S. P. Sigué, and G. Zaccour, "Dynamic cooperative advertising in a channel," Journal of Retailing, vol. 76, no. 1, pp.71-92, Spring 2000

[29] J. M. Lattin and R. E. Bucklin, "Reference effects of price and promotion on brand choice behavior," Journal of Marketing Research, vol. 26, no. 3, pp. 299-310, Aug. 1989.

[30] J. J. Ganuza, G. Llobet, and B. Dominguez, "R\&D in the pharmaceutical industry: A world of small innovations," Management Science, vol.55, no. 4, pp. 539-551, Apr. 2009.

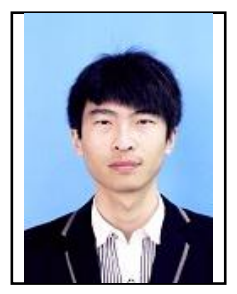

Baojun Thang received the B.S. degree in School of Management Engineering from Shandong Jianzhu University, Jinan, China in 2015. He is currently working toward the Master's degree with Business School, University of Shanghai for Science and Technology, Shanghai, China.

His research interests include decision analysis, supply chain management and risk management.

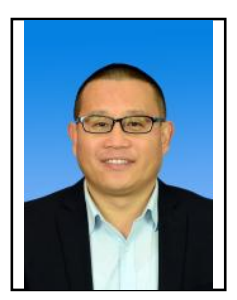

Shaojian Qu was born in China in 1978. He received the $\mathrm{PhD}$ in operational research from Xi' an Jiaotong University, Xi' an,China in 2008, and engaged in the Postdoctoral researches in Harbin Institute of Technology, Harbin, China and National University of Singapore, Singapore, from 2008 to 2011.

$\mathrm{He}$ is currently a professor with University of Shanghai for Science and Technology, Shanghai, China. His research interests are in decision analysis, game theory, supply chain management. Hehas published more than 30 papers in refereed journals such as European Journal of Operations Research, Journal of Optimization Theory and Applications, Computational Optimization and Applications, Journal of Global Optimization and Operations Research Letters.

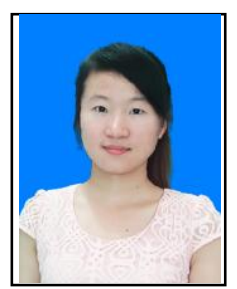

Panpan Li received the B.S. degree in economics and management college from West Anhui University, Lu'an, China in 2015. She is currently working toward the Master's degree with Business School, University of Shanghai for Science and Technology, Shanghai, China.

Her research interests include supply chain management, enterprise management and public management. 